\title{
PARTITIONS OF UNITY AND APPROXIMATION
}

\author{
C. DE BOOR AND R. DEVORE ${ }^{1,2}$
}

\begin{abstract}
We show that for certain translation invariant spaces $S$, a necessary and sufficient condition for the eventual denseness of the corresponding scaled spaces $S_{h}$ is that $S$ contain a stable and locally supported partition of unity. These results have been motivated by recent work on approximation by multivariate piecewise polynomials on regular meshes.
\end{abstract}

1. Introduction. We are interested in the existence of partitions of unity in certain translation invariant spaces. We are motivated by recent investigations (listed in the references) of approximation by multivariate piecewise polynomial functions on regular partitions-a partition $\tau$ of $\mathbf{R}^{m}$ is called regular if $j+\tau=\tau$ for all $j \in \mathbf{Z}^{m}$. These investigations study the degree of approximation from the spaces $\pi_{k}^{\rho}(h \tau)$, $h>0$, which consist of pp (i.e., piecewise polynomial) functions in $C^{\rho}\left(\mathbf{R}^{m}\right)$ of total degree $k$ on the dilated partition $h \tau$. It has been possible to determine the approximation order from these spaces only for very special partitions and certain values of $\rho$ and $k$. In particular, there are no general results which characterize when $\bigcup_{h>0} \pi_{k}^{\rho}(h \tau)$ is dense in the space of continuous functions with compact support.

The main technique for proving denseness or giving lower estimates for the degree of approximation has been to construct a partition of unity in $\pi_{k}^{\rho}(\tau)$ consisting of certain multivariate B-splines known as box splines and to use this partition of unity to construct suitable approximation schemes called quasi-interpolants.

It has not been clear to us whether this represents the only viable approach to the construction of suitable approximants. For example, in [BDH] we have asked the following:

Is $\bigcup_{h>0} \pi_{k}^{\rho}(h \tau)$ dense in $C_{0}\left(\mathbf{R}^{s}\right)$ if and only if $\pi_{k}^{\rho}(\tau)$ contains a good partition of unity?

By a good partition of unity we mean a (countable) collection $\Phi$ of functions with the properties that

(i) $\sup _{\phi \in \Phi}$ diam supp $\phi<\infty$;

(ii) $\sum_{\phi \in \Phi} \phi=1$;

(iii) $\sum_{\phi \in \Phi}|\phi|<\infty$.

Received by the editors May 4, 1984.

1980 Mathematics Subject Classification. Primary 41A15, 41A25.

Key words and phrases. Degree of approximation, partition of unity, piecewise polynomial.

${ }^{1}$ Both authors were sponsored by the United States Army under Contract No. DAAG29-80-C-0041.

${ }^{2}$ This material is based upon work supported in part by the National Science Foundation under Grant No. DMS-8320562. 
Actually, it is easy to see that the existence of a good partition of unity is sufficient for denseness. Namely, if $x_{\phi} \in \operatorname{supp} \phi$, then $L f:=\sum_{\phi \in \Phi} f\left(x_{\phi}\right) \phi$ gives an operator $L$ whose dilate $L_{h}:=\sigma_{1 / h} L \sigma_{h}$, with $\left(\sigma_{h} f\right)(x):=f(h x)$, provides an approximation $L_{h} f$ in $\pi_{k}^{\rho}(h \tau)$ which converges to $f$ as $h \rightarrow 0$.

It turns out, but is much more difficult to prove, that the existence of a good partition of unity is necessary for denseness. Here we shall present a proof of this fact for the univariate case but for much more general spaces $S$ than just pp ones, in an attempt to extract the essential features of $\mathrm{pp}$ function spaces on regular partitions.

The ideas of the proof seem to carry over to the multivariate case, but the constructions involved are much more involved. For this reason, we feel that it is beneficial to present here the basic ideas in their simplest setting and report elsewhere on their multivariate analogues.

2. The main results. We let $S$ be a space of functions on $\mathbf{R}$ with the following properties:

(i) $S$ is translation invariant, i.e., $f \in S \Rightarrow f(\cdot \pm 1) \in S$;

(ii) $\mu:=\left.\operatorname{dim} S\right|_{[0,1]}<\infty$;

(iii) $\operatorname{dim} S=\infty$;

(iv) $S$ is closed under uniform convergence on compact sets.

We note for later reference that (i) and (iii) imply

$$
\text { (v) }\left.\operatorname{dim} S\right|_{(0, \infty)}=\infty \text {. }
$$

These are the essential properties of pp spaces over regular meshes. However, it is easy to give examples of such spaces $S$ which are not pp. For example, if $\Phi$ is a finite collection of compactly supported functions, then the closure under uniform convergence on compact sets of the span of the translates $\phi_{j}:=\phi(\cdot-j), j \in \mathbf{Z}, \phi \in \Phi$, has these properties. The results of this section will show that any space $S$ satisfying (2.1) can be obtained this way modulo some finite-dimensional space.

Associated with $S$ we have the dilated spaces $S_{h}:=\sigma_{1 / h} S$.

THEOREM 1. If $\cup_{h>0} S_{h}$ is dense in $C_{0}(\mathbf{R})$, then $S$ contains a good partition of unity.

In preparation for the proof, we now construct certain compactly supported functions in $S$. Let $S^{-}$denote the subspace of $S$ consisting of those functions which vanish on $(-\infty, 0]$. It will follow later that $S^{-}$is nontrivial. For now, we prove

LEMMA 2. For each integer $n>0$, there is an $R_{n}$ such that any $\phi \in S$ vanishing on $\left[-R_{n}, 0\right]$ agrees on $[0, n]$ with some function from $S^{-}$.

Proof. For $r, j>0$, we let $L_{r, j}$ denote the space of functions defined on $[0, j]$ which are restrictions of functions in $S$ which vanish on $[-r, 0]$. Then $L_{r, j} \subseteq L_{r^{\prime}, j}$ whenever $r>r^{\prime}$. Hence $\left(\operatorname{dim} L_{r, j}\right)_{r=1}^{\infty}$ is a decreasing sequence of (nonnegative) integers and therefore is eventually constant. This implies that we can find an 
increasing sequence $\left(R_{j}\right)$ such that $L_{r, j}=L_{j}:=L_{R_{j}, j}$ for all $r \geqslant R_{j}, j=1,2, \ldots$ Now given $n>0$ and $\phi \in L_{n}$, we define

$$
n_{i}:=n+i, \quad r_{i}:=\max \left\{R_{n_{i}}, n_{i}\right\}, \quad i=0,1, \ldots
$$

Then, since $r_{1} \geqslant R_{n_{1}} \geqslant R_{n}$, there is an $f_{1} \in S$ which vanishes on [- $\left.r_{1}, 0\right]$ and agrees with $\phi$ on $[0, n]$. Similarly, $r_{2} \geqslant R_{n_{2}} \geqslant R_{n_{1}}$ and so there is an $f_{2} \in S$ which vanishes on $\left[-r_{2}, 0\right]$ and agrees with $f_{1}$ on $\left[0, n_{1}\right]$. In this manner, we obtain a sequence $\left(f_{j}\right)$ such that $f_{j}$ vanishes on $\left[-r_{j}, 0\right]$ and agrees with $f_{i}$ on $\left[0, n_{i}\right]$ for all $i<j$. Hence $f_{j}$ converges uniformly on compact sets to some $f$, necessarily in $S^{-}$because of (2.1)(iv), with the desired properties.

Now let $R:=R_{1}$ be the constant of Lemma 2 . We improve Lemma 2 in the following way:

Lemma 3. If $f \in S$ vanishes on $[-R, 0]$, then $\phi:=f \chi_{[0, \infty)}$ is in $S^{-}$.

Proof. From Lemma 2, we know an $f_{0} \in S^{-}$for which $f-f_{0}$ vanishes on $[-R, 1]$. Hence there is some $f_{1}$ with $f_{1}(\cdot+1) \in S^{-}$, therefore also $f_{1} \in S^{-}$, for which $f-f_{0}-f_{1}$ vanishes on $[-R, 2]$. In this manner, we construct a sequence $\left(f_{j}\right)$ in $S^{-}$ for which $f_{0}+\cdots+f_{j}$ converges to $\phi$. Since $S^{-}$is closed under uniform convergence on compact sets (by (2.1)(iv)), $\phi \in S^{-}$.

Next, we construct a basis of compactly supported functions for $S^{-}$. Recall the definition $\mu:=\left.\operatorname{dim} S\right|_{[0,1]}$ and set $M:=\mu R$.

LEMMA 4. If $f \in S^{-}$, then there is a function $\phi \in S$ which is supported on $[0, M]$ and agrees with $f$ on $[0,1]$.

Proof. Set $J:=[M, M+R]$ and consider the functions $f_{j}:=f(\cdot-j), j=$ $0, \ldots, M$. Since $\left.\operatorname{dim} S\right|_{J} \leqslant M$, these functions are linearly dependent on $J$. Hence there are numbers $c_{j}$, not all zero, such that $\psi_{0}:=\sum c_{j} f_{j}$ vanishes on $J$. Let $i$ be the smallest value of $j$ for which $c_{j} \neq 0$. Then $\psi:=\psi_{0}(\cdot-i) / c_{i}$ agrees with $f$ on $[0,1]$ and vanishes on $a+[-R, 0]$ with $a:=M+R-i$. Therefore, by Lemma 3 , $\psi_{1}:=\psi(\cdot+a) \chi_{[0, \infty)}$ is in $S^{-}$and so $\phi:=\psi-\psi_{1}(\cdot-a)$ has the desired properties.

It follows from Lemma 4 that there is a finite collection $B$ of functions supported on $[0, M]$ whose restrictions to $[0,1]$ form a basis for $\left.\left(S^{-}\right)\right|_{[0,1]}$. We use the abbreviation $b_{j}:=b(\cdot-j), b \in B, j \in \mathbf{Z}$. The peeling-off argument of Lemma 3 shows that the functions $b_{j}, b \in B, j=0,1,2, \ldots$, form a basis for $S^{-}$. But, in order to insure local linear independence of this basis, we need to choose $B$ with some care. Precisely, we choose $B$ so that length $(B):=\sum_{b \in B}$ length $(b)$ is minimal. Here, we mean by length $(b)$ the smallest integer $r$ for which supp $b \subseteq[i, i+r]$ for some integer $i$.

LemMa 5. For any interval $J:=[r, r+R]$, with $r$ an arbitrary integer and $R$ as before, the collection

$$
\Phi_{J}:=\left\{b_{j}: \operatorname{supp} b_{j} \cap J \neq \varnothing\right\}
$$

is linearly independent over J. 
Proof. Suppose that we have $f:=\sum_{\phi \in \Phi_{J}} c_{\phi} \phi=0$ on $J$. Then, by Lemma 3, we can find some $g \in S^{-}$so that $f_{1}:=f-g(\cdot-(r+R))$ vanishes on $[r, \infty)$. If not all the coefficients $c_{\phi}$ were zero, then we could choose some $\phi:=b_{j}$ with $c_{\phi}=: c_{b_{j}} \neq 0$ and so that $j$ is as small as possible. Since $B$ is linearly independent over $[0,1],\left.\left(f_{1}\right)\right|_{[j, j+1]} \neq$ 0 , and hence we must have $j<r$. Since $f_{1}$ vanishes outside $[j, r]$, while $\operatorname{supp} b_{j}$ intersects $[j, j+1]$ as well as $J$, this would imply that

$$
\text { length }\left(f_{1}\right) \leqslant r-j<\text { length }\left(b_{j}\right)
$$

while $f_{2}:=f_{1}(\cdot+j) \in S^{-}$and, on $[0,1]$, is in the span of $B$ with the coefficient of $b$ not zero. But then $B^{\prime}:=(B \backslash\{b\}) \cup\left\{f_{2}\right\}$ would also supply a basis and length $\left(B^{\prime}\right)<$ length $(B)$, contradicting our choice of a basis of minimal length.

Suppose now that $f=\sum c_{\phi} \phi$ with the sum over all $\phi=b_{j}$ with $j \in \mathbf{Z}$ and $b \in B$. Then the local linear independence just proved allows the conclusion that

$$
\left|c_{b_{j}}\right| \leqslant \text { const }\left\|\left.f\right|_{[j, j+R]}\right\|_{\infty}
$$

for some constant independent of $b$ and $j$.

Since $\left.S\right|_{[-R, 0]}$ is finite dimensional, we can choose a finite-dimensional subspace $T$ of $S$ which is carried faithfully onto $\left.S\right|_{[-R, 0]}$ by the restriction map $\left.f \mapsto f\right|_{[-R, 0]}$. For $s \in S$, let $s_{T}$ be the unique element of $T$ which agrees with $s$ on $[-R, 0]$. Then $\left(s-s_{T}\right) \chi_{[0, \infty]} \in S^{-}$by Lemma 3 , hence on $[0, \infty)$,

$$
s=s_{T}+\sum_{j \geqslant 0} \sum_{b \in B} c_{b_{j}} b_{j}
$$

for suitable coefficients $c_{b_{j}}$. Since $\left.S\right|_{[0, \infty)}$ is infinite dimensional by $(2.1)(\mathrm{v})$, this shows that $B \neq \varnothing$ and that $S^{-}$is also infinite dimensional. Further, since the linear space of all linear maps from $T$ to $\left.S\right|_{[-R, 0]}$ has dimension $(\operatorname{dim} T)^{2}$, the maps

$$
\left.T \rightarrow S\right|_{[-R, 0]}:\left.f \mapsto f(\cdot-a)\right|_{(-R, 0]}, \quad a \in A,
$$

must be linearly dependent whenever $A \subset \mathbf{Z}$ and $|A|>(\operatorname{dim} T)^{2}$. This together with Lemma 3 implies that, for any $A \subset \mathbf{Z}$ with more than $(\operatorname{dim} T)^{2}$ elements, there exist coefficients $c_{a}, a \in A$, so that

$$
\max c_{a}=\max \left|c_{a}\right|=1, \quad \chi_{[0, \infty)} \sum_{a \in A} c_{a} f(\cdot-a) \in S^{-} \quad \text { for all } f \in T .
$$

Proof of Theorem 1. Let $\tilde{g}$ be the continuous piecewise linear function with integer breakpoints which has the value 1 at $x=1,2,3$ and vanishes at all other integers. Since $\tilde{g} \in C_{0}(\mathbf{R})$, there is $\tilde{s}_{h} \in S_{h}$ such that $\tilde{s}_{h}$ converges to $\tilde{g}$ uniformly on R. From now on, we consider only $h \in 1 / \mathbf{N}$. We write $s_{h}:=\sigma_{h} \tilde{s}_{h}$ and $g_{h}:=\sigma_{h} \tilde{g}$. Then $\left\|g_{h}-s_{h}\right\| \rightarrow 0$ as $h \rightarrow 0$. Since $s_{h} \in S$, we have $s_{h}=t_{h}+s_{h}^{-}$on $[0, \infty)$, with $t_{h} \in T$ and $s_{h}^{-} \in S^{-}$. Now choose $A \subset 8 \mathrm{~N} / h$ as above. Then the functions $g_{h}(\cdot-a)$, $a \in A$, are supported in $[0, \infty)$ and have disjoint supports. We define

$$
G_{h}:=\sum_{a \in A} c_{a} g_{h}(\cdot-a)
$$

with the $c_{a}$ as given by (2.4). Then $f_{h}:=\sum_{a \in A} c_{a} s_{h}(\cdot-a)$ is in $S^{-}$, and $\left\|f_{h}-G_{h}\right\| \rightarrow 0$ as $h \rightarrow 0$. 
Now, because of (2.4), $G_{h}$ is identically one on an interval of the form [ $m_{h}-1 / h$, $\left.m_{h}+1 / h\right]$. Hence, with $F_{h}:=f_{h}\left(\cdot+m_{h}\right)$, we have $\left\|F_{h}-1\right\|_{[-1 / h, 1 / h]} \rightarrow 0$ as $h \rightarrow 0$. Since $f_{h} \in S^{-}$, we can write $F_{h}=: \sum_{j \in \mathbf{z}} \sum_{b \in B} c_{b_{j}}(h) b_{j}$. In view of (2.2),

$$
\left|c_{h_{i}}(h)\right| \leqslant \text { const for all } b, j, h \text {. }
$$

We can take therefore a subsequence $h^{\prime}$ of $h$ so that, for all $b$ and $j, c_{b_{1}}\left(h^{\prime}\right)$ converges as $h^{\prime} \rightarrow 0$ to some $c_{b_{i}}$. It follows that $\sum_{j \in \mathbf{Z}} \sum_{b} c_{b_{j}} b_{j}$ converges uniformly on compact sets to some function $F \in S$. Since we also have that $F_{h}$ converges to $F$ uniformly on compact sets, we must have $F=1$. Thus $\left(c_{b_{j}} b_{j}\right)_{b \in B, j \in \mathbf{Z}}$ is a partition of unity in $S$. It is good because of (2.5).

\section{REFERENCES}

[BD] C. de Boor and R. DeVore, Approximation by smooth multivariate splines, Trans. Amer. Math. Soc. 276 (1983), 775-785.

[BDH] C. de Boor, R. DeVore and K. Höllig, Approximation order from smooth pp functions, Approximation Theory. IV (C. Chui, L. L. Schumaker and J. Ward, editors), Academic Press, New York, 1983. pp. 353-357.

$\left[\mathbf{B H}_{1}\right]$ C. de Boor and K. Höllig, Bivariate box splines and smooth pp functions on a three-direction mesh, J. Comput. Appl. Math. 9 (1983), 13-28.

$\left[\mathbf{B H}_{2}\right]$. Approximation order from bivariate $C^{1}$-cubics: A counterexample, Proc. Amer. Math. Soc. 87 (1983), 649-655.

[DM] W. Dahmen and C. Micchelli, On the optimal order approximation rates for criss-cross finite element spaces, 1983.

[J] R.-q. Jia, Approximation by smooth bitariate splines on a three-direction mesh, Approximation Theory. IV (C. Chui, L. L. Schumaker and J. Ward, editors), Academic Press, New York, 1983, pp. $539-545$.

Mathematics Research Center, University of Wisconsin, Madison, Wisconsin 53705

Department of Mathematics, University of South Carolina, Columbia, South Carolina 29208 